\title{
An experimentally accessible probability of detection model for acoustic emission measurements
}

\author{
MARKUS G. R. SAUSE ${ }^{1}$, FLORIAN F. LINSCHEID ${ }^{1}$, MARKUS WIEHLER ${ }^{1}$ \\ ${ }^{1}$ University of Augsburg, Institute for Materials Resource Management, Mechanical Engineering, D-86135 \\ Augsburg
}

\begin{abstract}
In testing of structural components by the acoustic emission method, the requirement arises for quantitative prediction of the probability of detection (PoD) of an acoustic emission signal. Motivated similar as for other nondestructive testing methods, the suitability of given experimental settings to reach a certain likelihood of not missing relevant signals should be predicted. In contrast to other nondestructive testing methods, two of the key factors are not only the equipment and the inspector, but also the variability of the acoustic emission sources and the attenuation effects. As the strength of crack-based acoustic emission sources cannot be changed arbitrarily in the experiment, their characteristic amplitude distribution is accounted for by generation of reference datasets in small laboratory scale specimens. This assumes datasets with $100 \%$ PoD for those signals at a particular propagation distance. The prediction of the resulting PoD at another distance in a structure is achieved by means of amplitude reduction based on the measured attenuation values. For the latter, approaches using constant attenuation factors and attenuation mapping approaches are evaluated and compared to an experimental assessment of the PoD values using artificial test sources. Based on the agreement of calculated and measured PoD values, the presented approach appears promising to predict PoD values in geometrically and acoustically complex structures.
\end{abstract}

\section{Introduction}

In the field of nondestructive testing, probability of detection (PoD) concepts are frequently applied to compare different approaches and methods to evaluate a particular flaw type. The National Aeronautics and Space Administration (NASA) mainly developed that concept in the late 1960s and the early 1970s [1, 2]. Common for many nondestructive testing approaches is the aspect that the flaw to detect is purely passive. In general, the detectability for a specific flaw in a specific specimen investigated by a specific method is binary, i.e. true or false. In order to turn this into a statistical prediction, a large number of flaws and/or specimens and/or experimental settings are investigated. This provides the database for a statistical evaluation of the relevant factors of the detectability. The result of such studies is the PoD curve, usually visualized as the percentage of (a known) number of flaws detected vs. the flaw size. This is a step-function curve showing a more or less monotonous increase (as the flaw size decreases) until a saturation at $100 \%$ detected flaws is reached. The "flaw size" is a characteristic quantity relevant for the inspection procedure. Traditionally these are the dimensions of the flaw, but may readily be extended to account for other factors of influence, such as system gain at constant geometrical dimensions of the flaw.

This PoD concept became of widespread use as to its direct way of interpretation and because it is not only suitable to evaluate the performance of technical systems, but may also account for the analysis carried out by the inspector. As the final PoD curve is the result of all the individual contributions along the acquisition chain, it is possible to identify and distinguish these individual factors. Besides the influence of the human inspector, the evaluation algorithm, the acquisition system, the sensing system and the interplay between flaw and sensing field are key contributions to the final PoD. 
For the specific case of acoustic emission analysis, figure 1 provides an overview of these key contributions. Other than most of the nondestructive testing methods, the flaw in acoustic emission is not of passive nature. The source is usually considered as rapid displacement (e.g. due to crack growth) that causes emittance of a transient ultrasonic wave, which in turn is detected by sensors and then digitized using an acquisition board [3]. Due to this active source, the interpretation of the term "flaw size" is briefly revisited in this context. For the acoustic emission measurement, the acoustic energy release (strength) of the source and its orientation relative to the sensing system are the relevant quantities to consider. It is the matter of other approaches to translate these two factors into geometrical quantities like the geometrical "flaw size" of a crack (e.g. based on modeling work). Furthermore, the type of source might cause additional changes to the detectability even for similar geometrical "flaw size" [4]. In addition, the depth position may readily cause changes in the detectable signal strength at the surface level $[5,6]$.

Next, the propagation effects between source and sensor system affect detectability of an acoustic emission source. Regardless of the specific technical implementation of the acquisition chain, it is a requirement that the signal amplitude of the acoustic emission wave is distinguishable against the background noise floor. For all practical systems, signal attenuation will cause a reduction of amplitude as function of propagation distance to the sensor. Consequently, the overall PoD depends on the distance between the sensor system and the source. In addition, scattering of the wave field emitted by the source may cause additional reduction of signal amplitude or may cause areas of poor detectability due to shadowing effects. All of these factors so far are independent of the specific instrumentation and interpretation, so these are grouped together in figure 1 as items related to the test material and the structure tested.

Certainly, the choice of instrumentation will play a role in terms of the detectability. Depending on the chosen sensitivity, bandwidth and preamplifier gain, the acoustic emission sensor will be able to either detect the emitted signal or not detect it. Accordingly, the specific choice of sensor system may easily dominate the final PoD in those cases, when it becomes the bottleneck in the acquisition chain, e.g. because of poor sensitivity or because of limited bandwidth. Similarly, the subsequent acquisition chain will add its effects. This is highly specific for the particular approach taken, but breaks down for most of the commercial systems into the threshold used for detection, the trigger settings deciding on accepting / rejecting the signal and potential bandwidth limitations in the system electronics. Similar considerations may be made for threshold-free systems, e.g. streaming systems, where this step is simply moved to the offline step to decide when the signal is relevant or not. In addition, system electronics and software algorithms may further cause random (e.g. data leakage) or systematic (e.g. filter) reduction of the signals acquired. Together with the sensor system, this forms the instrumentation group seen in figure 1.

Finally, for all of the signals being available for analysis, data reduction steps are typically necessary. These involve manual or automated decisions to decide on the relevance of particular signals for the specific analysis. This is beyond the scope of this article, so it is not presented and discussed in detail. 


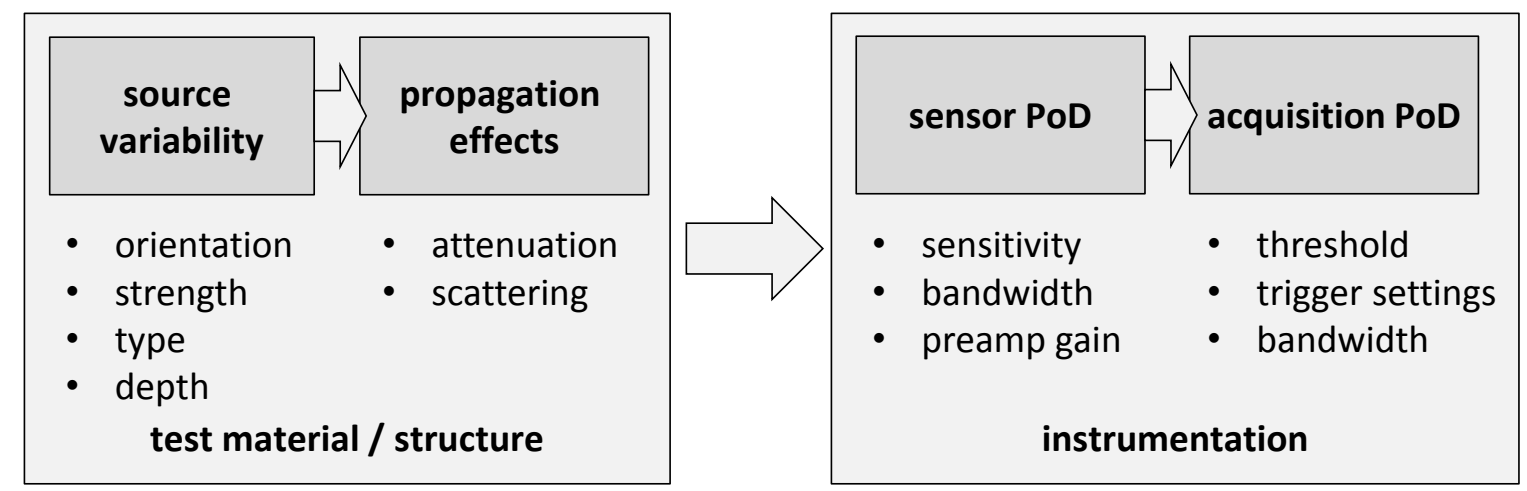

Figure 1: Schematic of factors influencing the probability of detection (PoD) in acoustic emission analysis.

\section{PoD concept for acoustic emission}

Inspired by the PoD approach proposed by Pollock et al. $[7,8]$ we decided to prepare a routine centered around an experimental approach to predict the PoD in a technical structure. For practical reasons it is impossible to validate a PoD approach for acoustic emission experimentally in the structure to be tested. Ultimately, this would require known test sources inside the test structure at known positions to check the number of detected signals vs. the number of released signals. Even if this would be possible in some cases (e.g. with embedded actuators) this is certainly not desirable for all test structures. Next, we limit ourselves to the detection of burst-type acoustic emission signals. For continuous acoustic emission the concept of isolated single signals is no longer valid and would require some fundamentally different approaches (definition of both types, see e.g. $[3,9,10]$ ).

Hence, we propose the following three steps to assess and predict the PoD for a test structure based on a combination of experimental data and model assumptions:

1) Record reference data set of burst-type acoustic emission sources at a known distance between source and sensor

2) Establish a signal propagation model for the structure under test

3) Perform PoD prediction based on the PoD model for the test structure considered

In the following, we elaborate the underlying assumptions of each step and discuss briefly the technical constraints.

\subsection{Reference data}

In $[7,8]$ it is proposed to account for the acoustic emission source variability by a mix of model considerations for fatigue crack growth and the respective model for acoustic emission release due to fatigue crack growth. This follows an ab-initio approach to predict the expected acoustic emission release in fatigue situations. For the procedure proposed here it suffices if such models are able to predict the acoustic emission amplitude distribution at given distance between source and sensor. Besides analytical routines, use of numerical methods may aid in this attempt $[9,11-13]$.

However, it is sometimes very difficult to translate the proposed concepts for other scenarios and load cases, especially when no proper model descriptions are available. Hence, we 
propose an alternative strategy to reach a reference dataset based on an experimental approach:

For the subsequent steps, all that is required is an amplitude distribution for all characteristic flaw types detected at a constant distance between source and sensor and at approximately $100 \%$ PoD. This requirement may sound inaccessible at first, but is feasible to do in practice, given some assumptions can be made.

First, the concise knowledge of distance between source and sensor requires precise localization of the events in an experimental setting with negligible influence of signal propagation effects (or similar propagation effects as expected in the test structure at a given distance). In practice, exclusion of all signals outside a narrow range of interest (e.g. few millimeters) returns only signals at approximately constant distance between source and sensor. Second, the selected detection settings (e.g. sensor and acquisition chain, software settings, filters and alike) should allow for a detection of approximately $100 \%$ of all acoustic emission sources at this distance. This requires operating the acquisition system at high gain, low threshold and very conservative filters. Likely, there are experimental conditions, where this is not easy to realize, but loss of signals at this stage would directly affect the calculated PoD later on, as this recorded dataset is further used as 100\% PoD reference. This assumption does partially neglect the aspect that specific acquisition settings may still result in a loss of detection of some acoustic emission signals. Concurrent concepts (such as signal streaming vs. signal triggering) may well lead to different number of signals for the same experiment. Similarly, extensive post-processing to detect signals hidden in the noise floor (e.g. [14]) will certainly lead to different number of signals, than a simple threshold based approach. Therefore, we would like to emphasize again that the reference dataset is assumed to be $100 \%$ PoD. Keeping the same evaluation settings for the reference dataset and the structure under test, this should still result in a similar effect of PoD reduction in both cases.

Furthermore, mixing the information of multiple experiments, multiple types of specimens and multiple sensors of the same type is good practice to ensure a minimum of bias in this reference dataset. The latter puts in another assumption, regarding the rule of mixture of different source types. Hence, some practical examples for generating reference datasets for fiber-reinforced materials are provided in section 3.1.

Due to the confinement effect in small samples versus large structures acoustic emission amplitudes are generally measured too large in amplitude in small scale samples [15]. Based on the observations of Hamstad et al., an experimental approach to assess the reduction in amplitude is to perform comparison of amplitudes in small-scale specimens and large structures. The resulting difference in amplitude (i.e. typically 5 to $13 \mathrm{~dB}$ ) can then be accounted for in equation (1) by increasing the detection threshold by this value. For the presented approach, this is identical to a decrease of the signal amplitudes of the reference dataset, but the increase in detection threshold is technically easier to implement.

\subsection{Signal propagation model}

As briefly discussed above, signal detectability will significantly suffer from wave attenuation and scattering. Similar as for the previous section, there are several possibilities how to account for this effect. In $[7,8]$, this step is accounted for using a generic attenuation model. For the metallic materials discussed therein, the effect of attenuation is well captured by implementation of an isotropic attenuation model assuming a linear attenuation coefficient $\alpha$. For anisotropic materials, such as fiber-reinforced materials, an attenuation coefficient as function of propagation angle $\alpha(\theta)$ is generally required. This could be obtained from model 
calculations (see e.g. [9]) or measurements. A conservative approach for a PoD could use the highest attenuation $\max (\alpha(\theta))$ and use this as an isotropic attenuation coefficient. However, many times the attenuation is not monotonous with distance. Therefore, a better approach is to obtain the attenuation "characteristics" from experimental measurements on the test structure (see section 3.2). This not only accounts for the material based attenuation including geometric spreading and guided wave formation, but also accounts for potential scattering along the propagation path. Finally, this concept can be extended to an attenuation mapping $\alpha(x, y, z)$. This requires test sources applied across the test structure to map the attenuation value to the $(x, y, z)$-position of the source. This data is readily available for source localization approaches using artificial neural networks [16], as this is inevitably required for their training stage.

\subsection{PoD model}

The final step consists of a suitable PoD model, able to turn the information of the two previous steps into a PoD prediction value. Assuming that the reference data is sufficient to describe the source variability and the propagation model is able to account for the propagation effects it should be possible to predict the detectable number of signals at a designated sensor position. To avoid an additional influence of the choice of instrumentation we make one key assumption:

The choice of instrumentation and the detection settings (e.g. sensor and acquisition chain, software settings, filters and alike) are not to be changed from the reference data measurement to the test structure measurement.

Consequently, we would expect the test structure data to suffer from the same instrumentation PoD as used in acquiring the reference data. Following this assumption, this reduces the task of PoD prediction to a prediction of those $A E$ signals, which arrive at the sensor position after travelling a certain distance.

To obtain the PoD value for a certain position on the test structure, the procedure is schematically presented in figure 2 and works as follows:

1) Define accept/reject criteria on the acquisition system side (typically simple threshold)

2) Apply attenuation mapping (or simplification thereof) to reference data $N_{\text {tot }}$

3) Evaluate the fraction of signals that the acquisition system failed to detect $N_{\text {failed }}$ (e.g. amplitude falls below threshold)

The PoD for this $(x, y, z)$ - location on the test structure evaluates using the number of detected signals $N_{\text {detected }}=N_{\text {tot }}-N_{\text {failed }}$ at sensor $S_{1}$ as:

$\operatorname{PoD}_{S_{1}}(x, y, z)=\frac{N_{\text {detected }}(x, y, z)}{N_{\text {tot }}}$

Usually, multiple sensors are attached to the test structure. From a general perspective, it suffices for the analysis process if only one sensor detects a signal. This does not allow analysis steps requiring signal arrival at multiple sensors (e.g. cross-correlation or source localization), but the source itself is detected by the acquisition system. Accordingly, the PoD for a particular $(x, y, z)$ - location evaluates as maximum PoD of each individual sensor out of the list of $K$ sensors $S_{1} \ldots S_{K}$ :

$\operatorname{PoD}(x, y, z)=\max \left(\operatorname{PoD}_{S_{i}}(x, y, z)\right) \forall i=1 \ldots K$ 

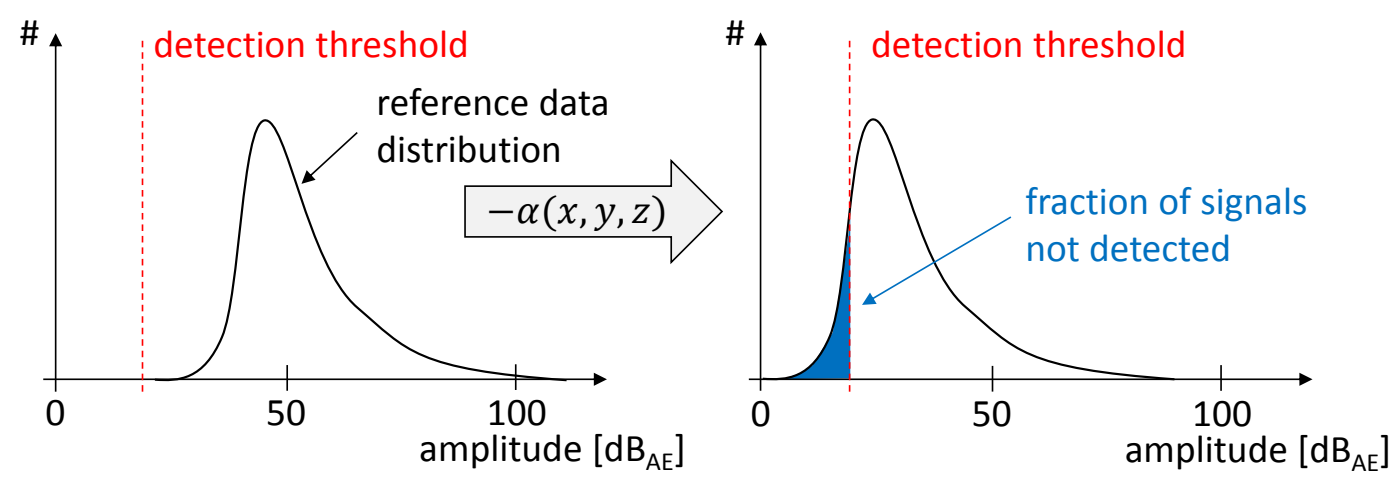

Figure 2: Proposed approach to account for PoD reduction due to attenuation.

\section{Experimental}

In order to demonstrate and validate our proposed concept, the experimental section first describes different approaches to obtain a reference dataset and then the procedure to measure attenuation. We present two case studies to demonstrate, how the PoD concept is applied to fiber-reinforced structures. The final step (PoD model) is then focus of section 4.

\subsection{Reference datasets}

For application to fiber-reinforced materials, it is very important to consider the various different source types that may happen in a test structure under load. For the purpose of this PoD analysis we propose to mix the information obtained from different mechanical tests, e.g. tensile tests, compressive tests, shear tests, fracture toughness tests. All data entering the reference dataset need to be acquired with instrumentation settings identical to those used later for the test structure and should be recorded for the same material as the test structure. For the example in figure 3, we used data of 10 samples for each experiment and stacking sequence, which were acquired in the conditions with test standards as listed in table 1. Details of the typical AE instrumentation for each of these cases can be found in earlier work $[9,17,18]$ and is hence not discussed in detail in the following.

\begin{tabular}{|l|l|l|l|c|}
\hline Name & Standard & \multicolumn{2}{|l|}{ Test condition } & Statistics \\
\hline Tensile test & $\begin{array}{l}\text { DIN EN ISO } \\
527\end{array}$ & $\begin{array}{l}\text { Unidirectional } \\
\text { (load axis parallel } \\
\text { to fiber axis) }\end{array}$ & $\begin{array}{l}\text { Cross- } \\
\text { ply }\end{array}$ & 20 \\
\hline V-notched rail shear test & ASTM D 7078 & $\begin{array}{l}\text { Unidirectional } \\
\text { (load axis } \\
\text { perpendicular to } \\
\text { fiber axis) }\end{array}$ & $\begin{array}{l}\text { Cross- } \\
\text { ply }\end{array}$ & 20 \\
\hline End-notched-flexure test & & Unidirectional & & 10 \\
\hline Double-cantilever-beam test & & Unidirectional & & 10 \\
\hline 4-point bending test & DIN EN ISO & $\begin{array}{l}\text { Unidirectional } \\
\text { (load axis parallel } \\
\text { to fiber axis) }\end{array}$ & $\begin{array}{l}\text { Cross- } \\
\text { ply }\end{array}$ & 20 \\
\hline Sum & 14125 & & 80 \\
\hline
\end{tabular}

Table 1: Summary of test conditions used to define reference dataset. 
For the present study, the instrumentation consists of WD type sensors, attached to a 2/4/6 preamplifier with $40 \mathrm{~dB}$ gain and $\mathrm{PCl}-2$ acquisition cards (all Mistras). An analog bandpass was included in the preamplifiers ranging from $20 \mathrm{kHz}$ to $1200 \mathrm{kHz}$. Acquisition was made based on a $35 \mathrm{~dB}_{\mathrm{AE}}$ threshold value at $10 \mathrm{MSP} / \mathrm{s}$ sampling rate with 10/80/300 (PDT/HDT/HLT) trigger settings. Data reduction at approximately $50 \pm 5 \mathrm{~mm}$ distance between source and sensor was carried out after localizing acoustic emission events with $\Delta \mathrm{t}$-based algorithms and sensor geometry corresponding to the respective experiment (see examples in $[9,17,18]$ for implementation).

For the five different experiments listed in table 1, the way of mixing the single datasets together puts some bias into the amplitude distribution of the reference dataset. Assuming some of the tests result in higher amplitude acoustic emission signals (on average) than other tests, it is important to consider the number of signals taken from each test. As some of the tests exhibit several orders of magnitude higher number of signals (e.g. Double-cantileverbeam test) than others (e.g. tensile test), the latter would not be statistically representative. Hence, we decided to first mix all datasets of the same test condition (i.e. all 10 measurements after filtering to $50 \pm 5 \mathrm{~mm}$ distance) and then select a random subset of 1000 signals from this mixed dataset. As next step, we then combined the 1000 signals of each experimental setting into one reference dataset (now holding 8000 signals), thus giving equal weight to each experimental configuration (laminate layup and load condition). An example of such a distribution is given in figure 3 -a.

However, there is an obvious bias inherent to this approach. There is no guarantee, that the later test structure will show a source distribution that is equal to the mix used for the reference dataset. In addition, the list of potential experiments to combine for the reference dataset is open ended, as more stacking sequences and test conditions could be added. Consequently, an alternative approach would consist in selecting the test case with the lowest amplitude values as refeđrence dataset, as this would be the most conservative choice. Another alternative is to focus only on a particular failure mode (e.g. tensile failure) and calculate PoD only for this failure mode scenario.

However, for a general situation, without knowing the potential mode of failure and likely occurrence of mixed failure modes, we are convinced that a mix of different experimentally obtained datasets is the most unbiased approach to calculate for the acoustic emission PoD.

In order to demonstrate the proposed PoD concept without additional bias in the reference dataset another approach will be used in this study. As it is hard to induce natural acoustic emission sources with controlled amplitudes in a structure, a piezoelectric pulser is used instead in order to generate test signals with varying amplitudes. As seen in figure 3-b, we apply a relatively smooth source distribution for this purpose. To generate a test signal, we use a conical piezoelectric element with brass backing as used in [19] as pulser system. The piezoelectric material is driven by an Agilent 33210A arbitrary waveform generator using a step function pulse with 20 ns edge time and hold times of $5 \mathrm{~ms}$ to avoid initiation of another acoustic emission within the observation window, i.e. the acquisition settings were chosen accordingly to ensure the second signal falls in the lock-out time of the measurement system. To reproduce the distribution of figure 3-b, an arbitrary number of 2258 signals are excited at each position with varying maximum signal voltage. We choose a signal amplitude high enough to yield a $100 \%$ PoD at distances close to the pulser. For statistical reasons, the experiment is repeated five times at each position. To facilitate a good coupling, we use Korasilone grease (Bayer) to compensate effects of dry contacts or entrapped air gaps at the 
contact interface. Details of the geometrical arrangement between the pulser and sensor are provided in section 3.3 and 3.4 for each of the test conditions.

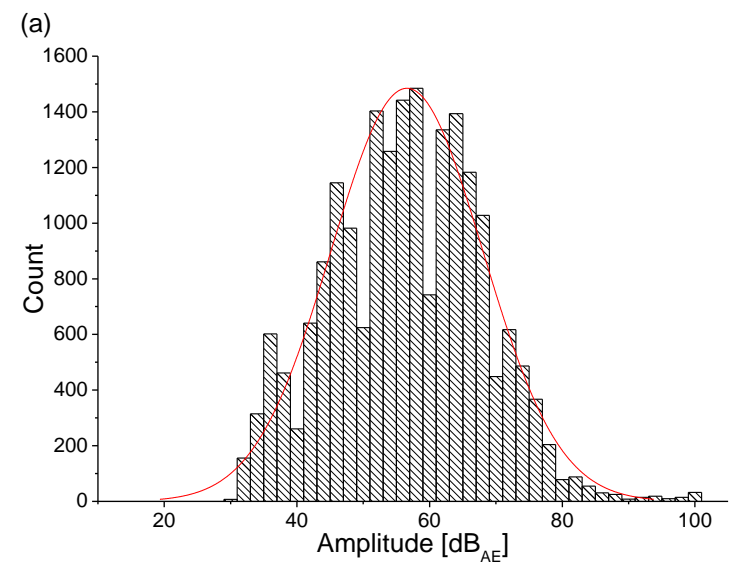

Figure 3: Realistic reference dataset (a) and smooth distribution used for concept validation (b)

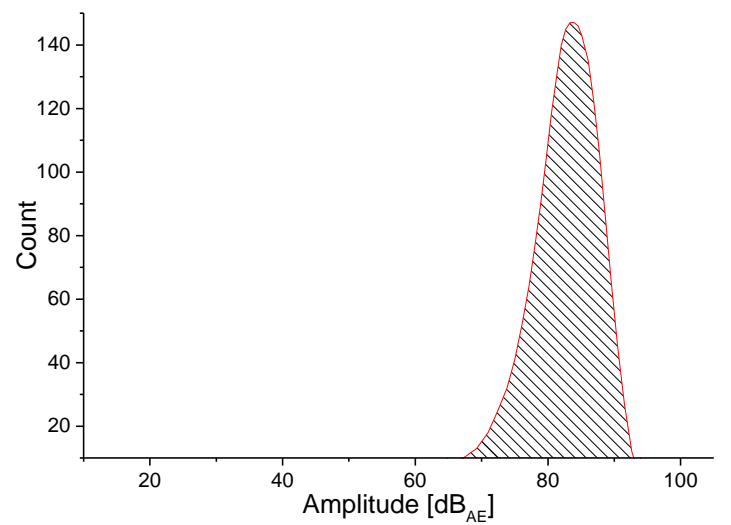

(b).

\subsection{Attenuation measurement}

In addition to the reference dataset, a measure of signal attenuation in the structure is required. As experimental approach, we used a WD type sensor, attached to a 2/4/6 preamplifier with $40 \mathrm{~dB}$ gain and $\mathrm{PCl}-2$ acquisition card (both Mistras). An analog bandpass was included in the preamplifiers ranging from $20 \mathrm{kHz}$ to $1200 \mathrm{kHz}$. Acquisition was made based on a $75 \mathrm{~dB}_{\mathrm{AE}}$ threshold value at $20 \mathrm{MSP} / \mathrm{s}$ sampling rate in synchronized acquisition mode (all channels trigger at threshold crossing at one sensor). The sensor position was kept constant and test signals were generated at distinct positions using a Hsu-Nielsen source according to ASTM E 976. Five repetitions were made at each designated position. Distances were chosen starting close to the sensor and then incrementing $20 \mathrm{~mm}$ typically until the signal was barely detected, or the outer dimensions of the test structure were reached. Figure 4-a shows an example of the measurement result of a cross-ply plate along $0^{\circ}, 45^{\circ}$ and $90^{\circ}$ direction. The absolute values of signal amplitudes of figure 4-a depend on the coupling quality of the test sensor, so these are not useful for the evaluation of the attenuation factors. Instead, the attenuation factors were determined from linear regression of the slopes of figure 4-a as $-8.9 \mathrm{~dB} / \mathrm{m}$ for $0^{\circ}$ propagation direction, $-4.9 \mathrm{~dB} / \mathrm{m}$ for $45^{\circ}$ propagation direction and $-6.3 \mathrm{~dB} / \mathrm{m}$ for $90^{\circ}$ propagation direction.

As alternative approach to provide a measure of attenuation, we evaluated the attenuation map for the test cases of section 3.3 and 3.4. The final result for the example of section 3.4 is shown color-coded in figure 4-b. To measure the attenuation map, we used four WD sensors attached to the structure at the designated positions of figure 4-b. Test signals were generated at distinct positions using a Hsu-Nielsen source according to ASTM E976. Acoustic emission signals are recorded using a 2/4/6 preamplifier with $40 \mathrm{~dB}$ gain and $\mathrm{PCl}-2$ acquisition card (both Mistras). An analog bandpass was included in the preamplifiers ranging from $20 \mathrm{kHz}$ to $1200 \mathrm{kHz}$. Acquisition was made based on a $75 \mathrm{~dB}_{\mathrm{AE}}$ threshold value at $10 \mathrm{MSP} / \mathrm{s}$ sampling rate in synchronized acquisition mode. For each of the attached WD sensors (channel 1 in figure 4b) it is possible to calculate the reduction of amplitude in $\Delta d B_{\mathrm{AE}}$ as function of the $(x, y, z)$ position when relating the test signal $\mathrm{dB}_{\mathrm{AE}, i}$ to the maximum of the series $\mathrm{dB}_{\mathrm{AE} \text {,max }}$ as: 
$\Delta \mathrm{dB}_{\mathrm{AE}}=\mathrm{dB}_{\mathrm{AE}, \max }-\mathrm{dB}_{\mathrm{AE}, i}$

As seen in figure 4-b, this allows to "map" inhomogeneous attenuation values and allows to improve the accuracy of the PoD prediction in a given test case as will be discussed in section 4.

(a)

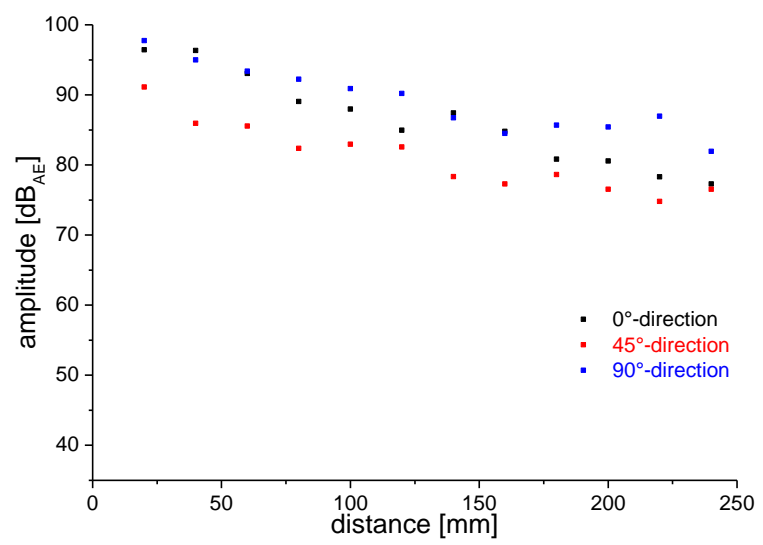

(b)

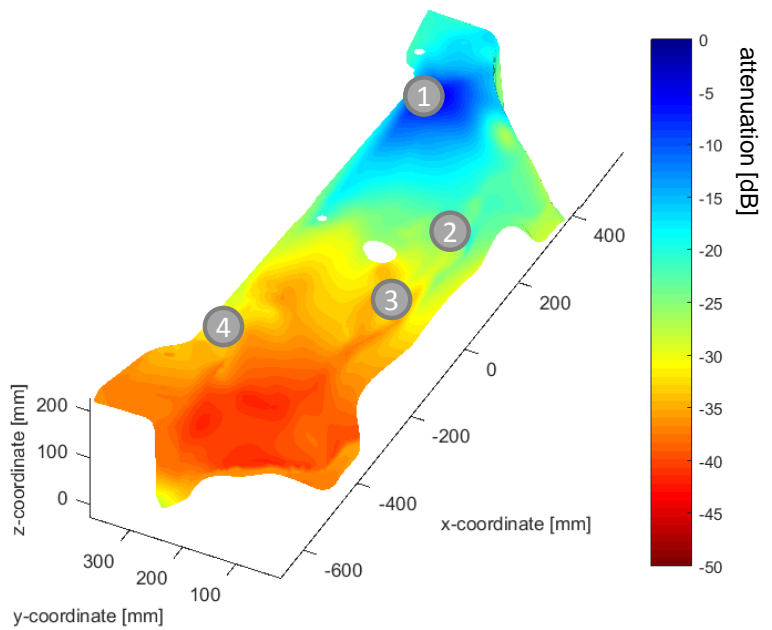

Figure 4: Result of attenuation measurement on a cross-ply plate in $0^{\circ}, 45^{\circ}$ and $90^{\circ}$ direction (a) and attenuation map of fiber-reinforced structural part for channel 1 (b).

\subsection{Case study 1 - Fiber reinforced plates}

In order to demonstrate the feasibility of the proposed PoD prediction approach, we decided to use two case studies for the comparison of experimental results to the PoD prediction. As first case study, we use a set of three carbon fiber / epoxy plates made from the prepreg system Sigrafil CE1250-230-39 with a size of $570 \mathrm{~mm} \times 570 \mathrm{~mm}$ and a thickness of $1.3 \mathrm{~mm}$. As stacking sequences, unidirectional $\left[\mathrm{O}_{3}\right]_{\text {sym }}$, cross-ply $\left[0 / \mathrm{OO}_{2}\right]_{\text {sym }}$ and quasi-isotropic $[0 / 60 /-60]_{\text {sym }}$ were used. In the notation used, subsets indicate repeated layers and "sym" indicates symmetry at the plate midplane. All plates were cured according to the material supplier's recommendation. For the experimental assessment of the PoD, independent measurements were made along the $0^{\circ}, 45^{\circ}$ and $90^{\circ}$ direction of each plate. For these measurements, the piezoelectric pulser is placed at the centre position of the plate and the sensor is moved to several distances ( $20 \mathrm{~mm}$ increments, details see schematic in figure 5). Each position of the sensor was checked five times, applying the settings and distribution described in section 3.1. Acoustic emission signals were recorded by a WD type sensor attached to a 2/4/6 preamplifier at $40 \mathrm{~dB}$ gain and a $\mathrm{PCl}-2$ acquisition card (both Mistras). An analog bandpass was included in the preamplifiers ranging from $20 \mathrm{kHz}$ to $1200 \mathrm{kHz}$. Acquisition was made based on a $25 \mathrm{~dB}_{\mathrm{AE}}$ threshold value at $10 \mathrm{MSP} / \mathrm{s}$ sampling rate with 20/200/700 (PDT/HDT/HLT) trigger settings.

\subsection{Case study 2 - Fiber reinforced structural part}

For proper exploration of the predictive capabilities of the approach, we decided to use a realistic structural composite part for case study 2 . To this end, we apply the same concept as described for the three plates to a structure with steep curvature changes, thickness variations, cutouts and realistic complexity of the stacking sequence. As the latter changes 
throughout the structural part, it is not feasible to provide a global stacking sequence, but overall it consists of fiber directions along $\pm 45^{\circ}, 90^{\circ}$ and $0^{\circ}$ as well as interspersed fabric layers. The part is fabricated using a carbon fiber epoxy system following a curing cycle in accordance with the material supplier's recommendations. As indicated in figure 5-b, the piezoelectric pulser was kept at constant position and the sensor distance was changed along four different directions relative to the pulser at $20 \mathrm{~mm}$ increments. Acoustic emission signals were recorded by a WD type sensor, attached to a 2/4/6 preamplifier with $40 \mathrm{~dB}$ gain and $\mathrm{PCl}-2$ acquisition card (both Mistras). An analog bandpass was included in the preamplifiers ranging from $20 \mathrm{kHz}$ to $1200 \mathrm{kHz}$. Acquisition was made based on a $25 \mathrm{~dB}_{\mathrm{AE}}$ threshold value at 10 MSP/s sampling rate with 20/200/700 (PDT/HDT/HLT) trigger settings.

(a)

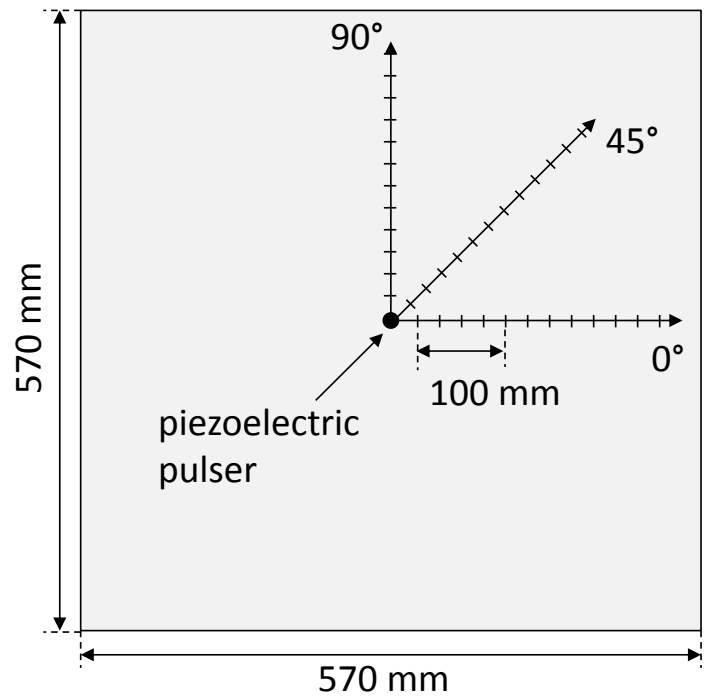

(b)

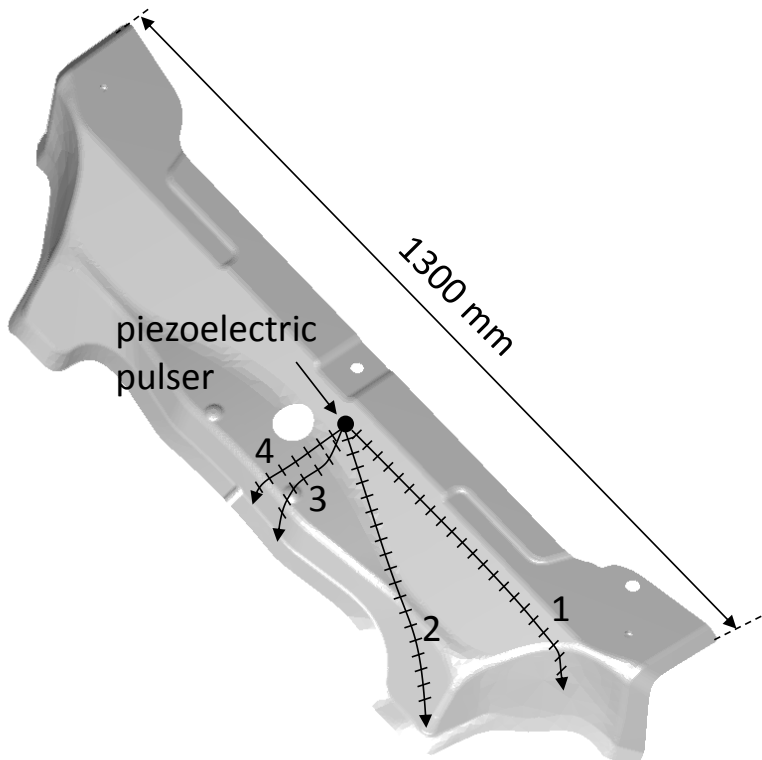

Figure 5: Schematic of PoD measurements in case study 1 (a) and in case study 2 (b).

\section{Results}

In the following, we present the results of the PoD measurements and discuss these relative to model calculations as obtained by our proposed method. This is first demonstrated using a quadratic fiber reinforced plate with various stacking sequences, then is demonstrated in application to a fiber reinforced structural part with reasonably complex attenuation characteristic.

\subsection{Case study 1 - Fiber reinforced plates}

Based on the experimental approach described in section 3.3, measurements of the number of detected signals as function of distance to the sensor position were carried out along three principal directions of each plate. In figure 6 -a, the measured signal amplitude distribution is shown for three distances to the sensor. For the cross-ply plate at $0^{\circ}$ direction of propagation, at $40 \mathrm{~mm}$ distance, still almost all signals were detected, i.e. 2218/2258 were recorded. Accordingly, the measured PoD evaluates as $98.2 \%$. At $100 \mathrm{~mm}$ distance, the less intense signals approach the detection threshold and partially fall below the value of $25 \mathrm{~dB}_{\mathrm{AE}}$, as seen by the significant left-shift of the amplitude distribution. At $240 \mathrm{~mm}$ a significant number of signals fall below the threshold, so that the PoD evaluates as $28.2 \%$ only. This is also seen in the reduced height of the signal distribution. The shape of the distribution curve of all three 
cases follows the shape of the input signal distribution (cf. figure 3-b). The full evaluation in terms of experimental PoD data is shown in figures 6-b, 6-c and 6-d for the cross-ply plate in propagation direction $0^{\circ}, 45^{\circ}$ and $90^{\circ}$. The dotted data represent the measurement results.

Superimposed to the measured POD values, two approaches of model calculations are presented. For each propagation direction, the solid line represents the approach using a constant attenuation factor as described in section 3.2. In addition, the dashed line represents the same PoD approach using the attenuation mapping data selected along the specific propagation directions. For the $0^{\circ}$ - and $90^{\circ}$ direction of propagation, there is good agreement between the experimental data and either of the two calculated approaches. Generally, the constant attenuation factor results in a smooth fall-off, as there is no change in the attenuation values as function of distance. In contrast, the attenuation mapping approach results in more changes of slope, as local deviations in the attenuation behavior are taken into account. This difference is seen best for the $45^{\circ}$ direction of propagation. In this case, the constant attenuation factor would significantly overestimate the real PoD. Using the attenuation mapping approach, the sudden drop of PoD at $75 \mathrm{~mm}$ and the evolution thereafter is captured well. In general, the discrepancy between the experimental data and the calculated results of the attenuation mapping approach is mostly due to the number of points used to establish the attenuation map, which is still much better than a constant attenuation value for each propagation direction.

(a)

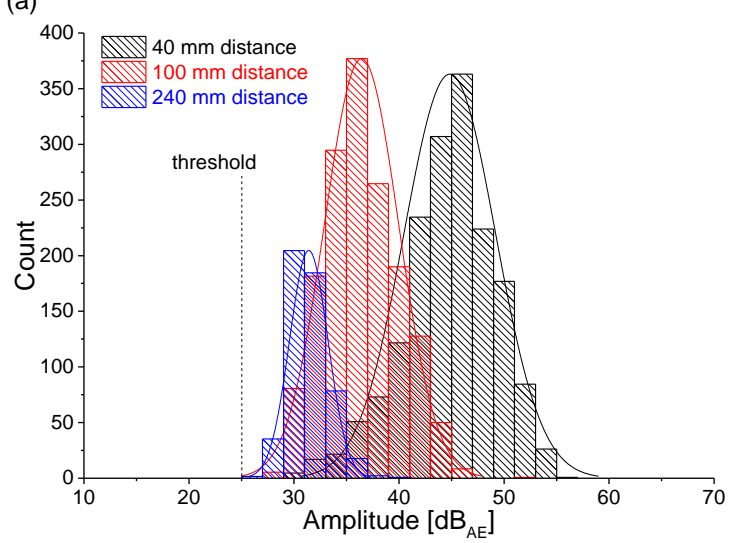

(c)

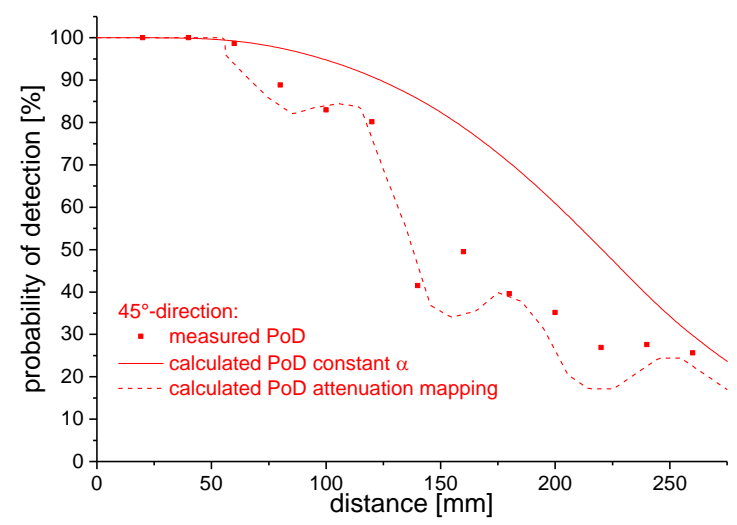

(b)

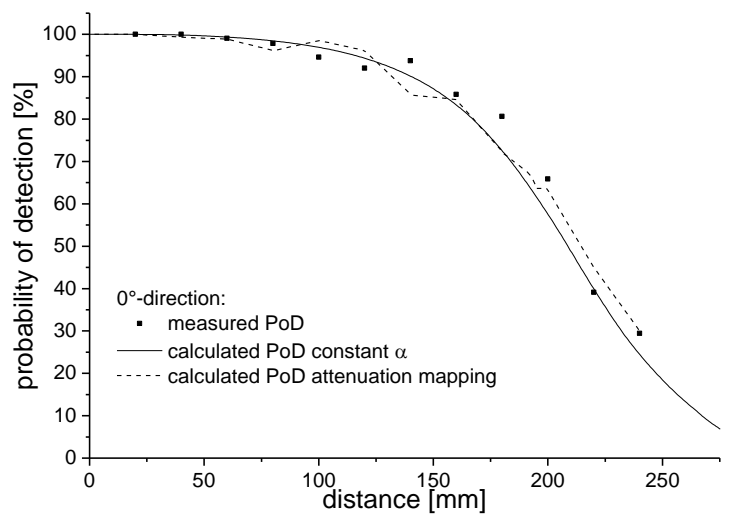

(d)

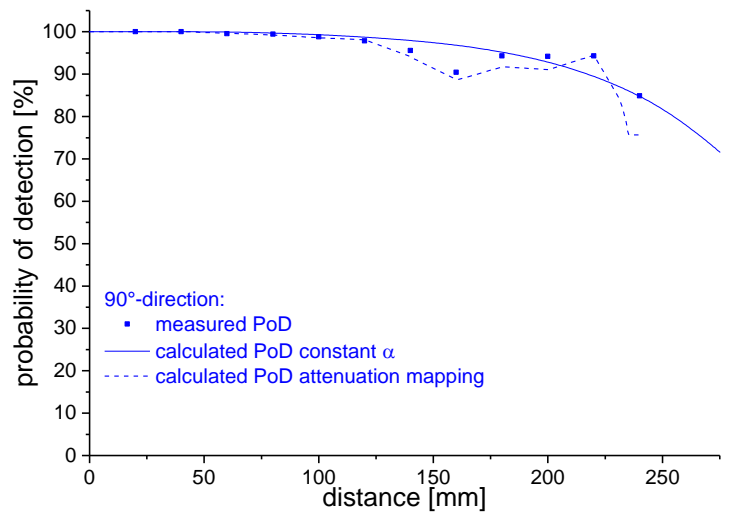

Figure 6: Typical amplitude distribution of acoustic emission signals detected at $40 \mathrm{~mm}$, $100 \mathrm{~mm}$ and $240 \mathrm{~mm}$ at $0^{\circ}$ orientation (a) as well as measured and computed PoD along $0^{\circ}$ (b), $45^{\circ}$ (c) and $90^{\circ}$ (d) direction for the cross-ply plate.

For practical applications, the PoD curves may be turned into a color-coded representation of POD values as function of position. Such information is provided in figure 7 for the quasi- 
isotropic plate. The visualization requires two pieces of information besides the sensor position. A measure for the attenuation in the structure and a relationship between PoD and distance to sensor. In the approach of figure 7-a the quasi-isotropic plate assumes the worst case attenuation value of $-9.1 \mathrm{~dB} / \mathrm{m}$ measured for this case and uses a corresponding PoDcurve of the quasi-isotropic plate (similar to figure 6-b, 6-c and 6-d). Given the a-priori knowledge of directional dependency of attenuation values for different propagation directions as discussed above, this may seem too simplistic. However, this approach represents the typical scenario for a more complex technical structure, where proper evaluation of the attenuation values as function of angle is impossible, as this may easily depend on the position of detection. As second approach, the attenuation map is used to visualize the corresponding PoD values in figure 7-b. For better comparability, both figures use a common color scale to represent PoD values in the range of $0 \%$ to $100 \%$. For the attenuation mapping approach of figure 7-b, the values measured in 11 rows and 11 columns (121 in total) are interpolated to yield a more uniform rendering. Obviously, the PoD values calculated using the attenuation map take into account the inhomogeneity of the propagation medium, as there are strong differences of the resulting PoD not solely as function of distance to the sensors. This is owed to different fiber orientations, as well as inhomogeneous fiber distribution and voids present in the test laminates. In addition, spots of low PoD are predicted for different locations than in the approach with constant attenuation. Based on the good agreement of this PoD prediction with the measured PoD values this is considered a more realistic view of the actual situation.

(a)

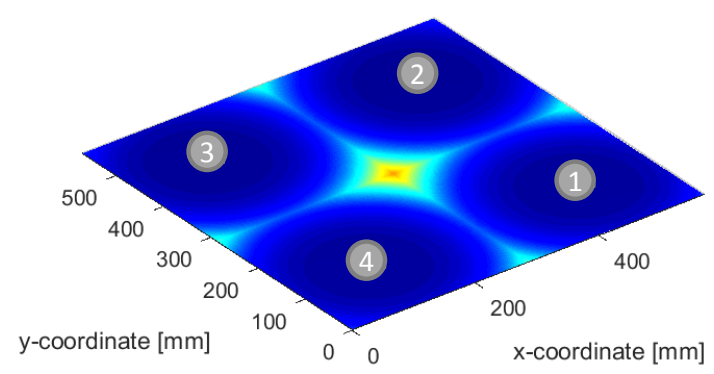

(b)

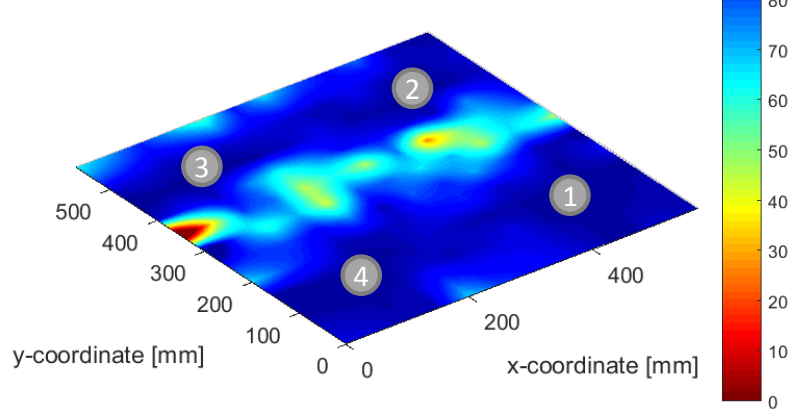

Figure 7: Comparison of resulting PoD for a scenario with four sensors applied on a quasiisotropic plate, assuming a constant attenuation profile of the worst case measured in $0^{\circ}$ direction (a) and using the attenuation mapping approach (b).

\subsection{Case study 2 - Fiber reinforced structural part}

As second case study, we present results from a fiber-reinforced structure with reasonable geometrical and acoustical complexity (cf. section 3.4). Similar as for the case study before, we evaluate the approach to use a constant attenuation factor and the approach using the attenuation mapping. As seen in figure 5, experimental datasets were collected along four different tracks on the surface of the structure (cf. figure 5 for position). From attenuation measurements we evaluated values of $-4.1 \mathrm{~dB} / \mathrm{m}$ (track 1), $-5.9 \mathrm{~dB} / \mathrm{m}$ (track 2), $-10.7 \mathrm{~dB} / \mathrm{m}$ (track 3) and $-10.4 \mathrm{~dB} / \mathrm{m}$ (track 4) using a linear regression approach.

For the experimental data it is worthwhile to note that the falloff generally does not have a smooth tendency, but has several ups and downs as function of distance to the test sensor. 
This is due to the different cutouts of the structure as well as specific changes in laminate thickness and stacking sequences at designated positions. This is especially pronounced for track 2, as the laminate sequence changes three times along the selected direction. Accordingly, three different regions and transition zones are observed in the measured PoD curves. The simplified approach to apply constant attenuation values leads to fairly smooth PoD curves, which are superimposed relative to the experimental data points as solid lines in figure 8. Except for figure 8-c the agreement with the experimental data is relatively low. The measured PoD values are either under- or overestimated. Especially for track 1 seen in figure 8-a, the calculated PoD overestimates the measured values significantly.

In contrast, the PoD values of the attenuation mapping approach match the measurement values much better. Based on the concept of the approach this is not unexpected, as it is possible to take into account the inhomogeneous attenuation values. Except for the first part of track 2 , this generally leads to a reasonably good description of the measurement values.

(a)

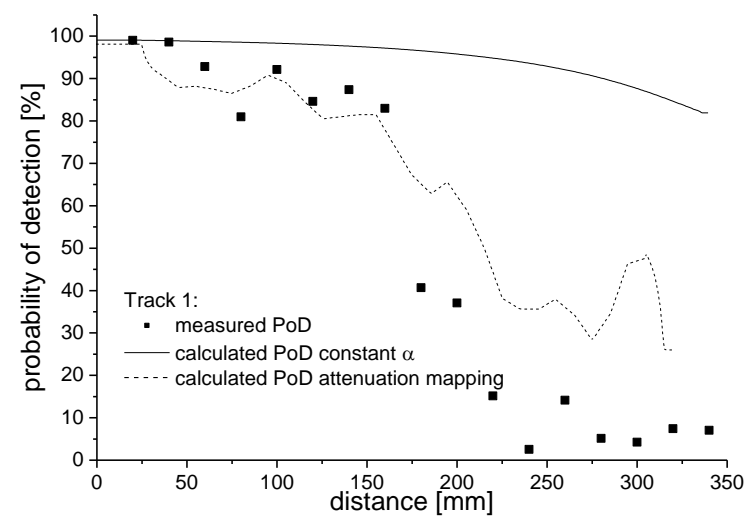

(c)

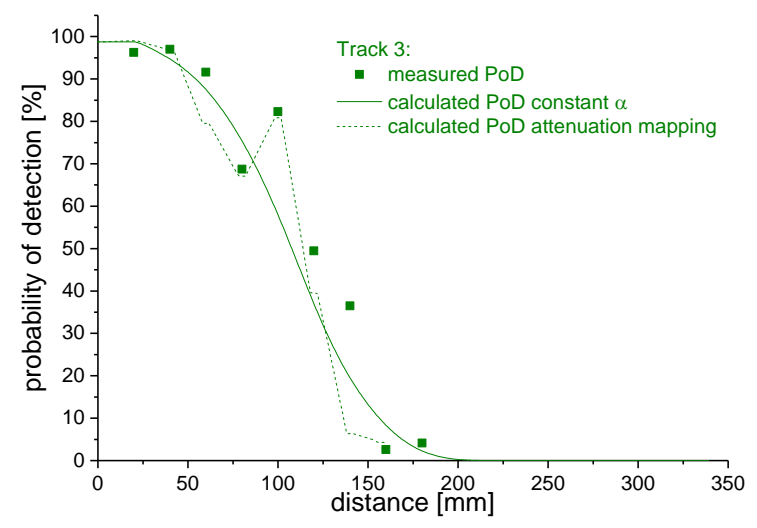

(b)

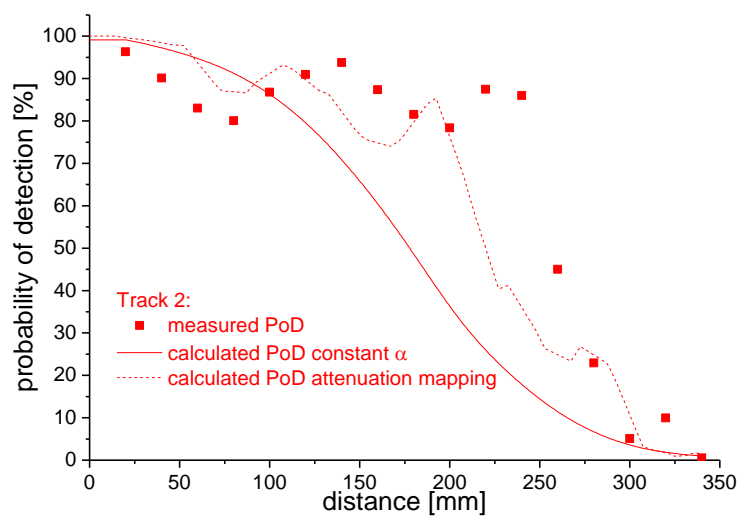

(d)

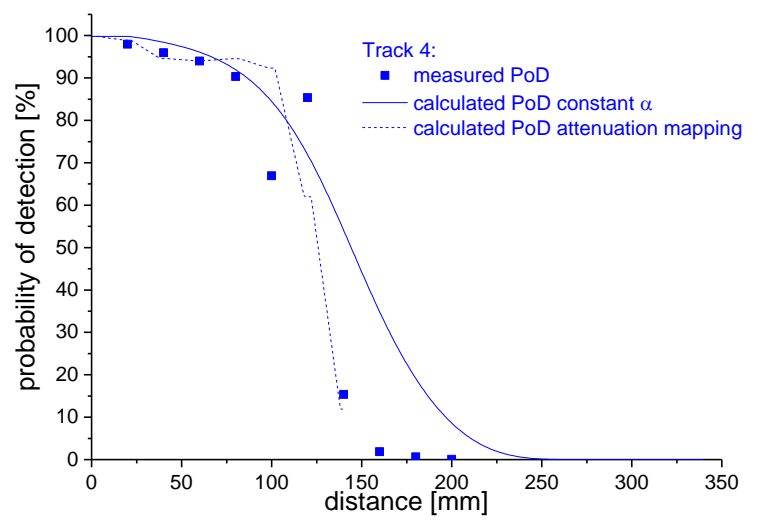

Figure 8: Measured and computed PoD for the four different tracks on structural part (see figure 5), track 1 (a), track 2 (b), track 3 (c) and track 4 (d).

Considering the same visualization approach as for case study 1, the aim of figure 9 is to contrast a simple approach with constant, isotropic attenuation values and the approach using attenuation mapping. Taking into account the conservative attenuation measure of $-10.7 \mathrm{~dB} / \mathrm{m}$, the resulting PoD values in the structure solely depend on the distance to the sensor as seen in figure 9-a. In the given situation this indicates that large parts of the structure reach PoD values close to zero. For the attenuation mapping approach presented in figure 9$b$, the PoD values are based on a measurement grid of 190 equally distributed test points, which are interpolated to yield a smoother representation. Based on the high attenuation 
value chosen for the PoD calculation of figure 9-a, the real detectability of acoustic emission sources is highly underestimated. The PoD calculation of figure 9-b reveals that a large part of the structure still has PoD values above $50 \%$. Accordingly, the proper evaluation in this case not only provides a more realistic view on the expected PoD values as function of position, but also allows the selection of an appropriate sensor network density.

(a)

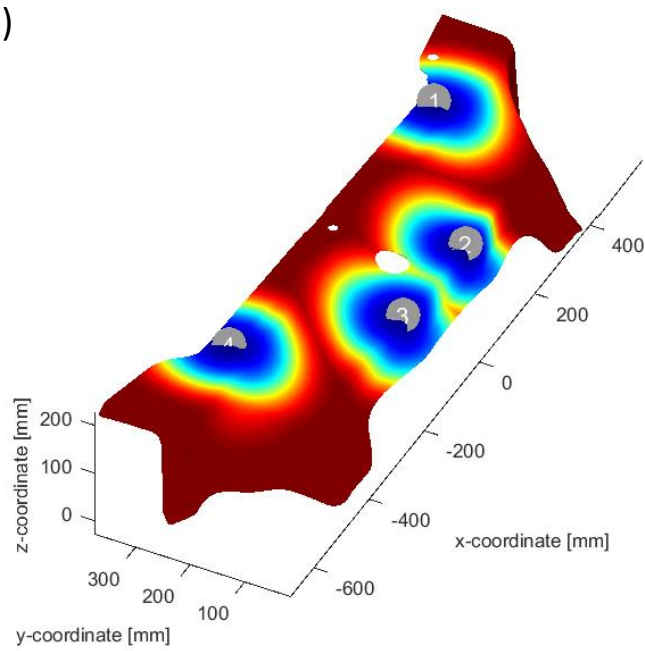

(b)

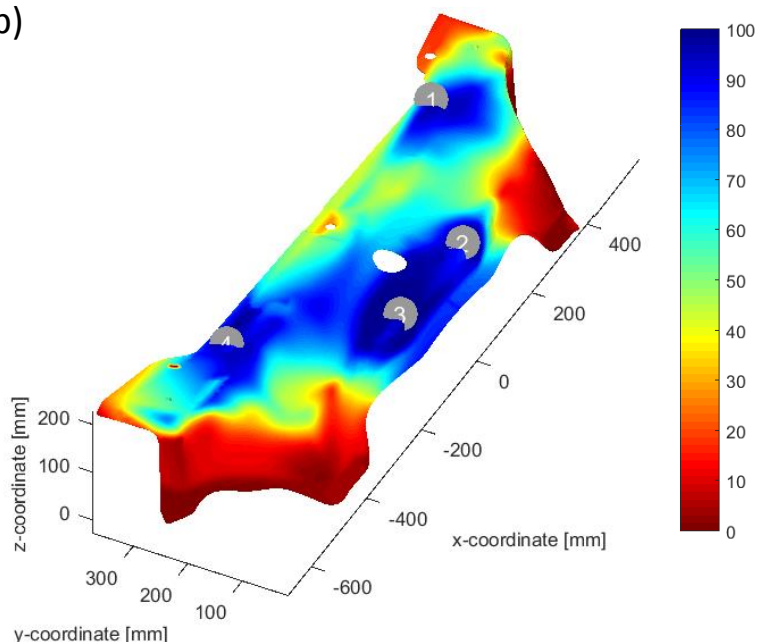

Figure 9: Comparison of resulting PoD for a scenario with four sensors on the test structure, assuming a constant attenuation profile of the worst case measured in track 3 (a) and using the attenuation mapping approach (b).

\section{Conclusion}

The presented approach for probability of detection calculation considers an experimentally obtained reference dataset, which appears more accessible than a theoretical, model based approach. The strength and weaknesses of this choice are briefly contrasted in table 2 below. The calculus of detectable signals at a given distance was demonstrated using two different approaches using experimental measurements of attenuation as basis. As conservative approach, the maximum attenuation coefficient found in the test structure may be applied, while less conservative, yet more accurate prediction is expected for attenuation mapping approaches, which was validated against an independent experimental approach. As the presented approach seems properly established, the predictive capabilities are intrinsically linked to the proper choice of the reference dataset.

For some applications, it might even be interesting to use different philosophies than the approach presented in section 3 . In some cases, it might be relevant to calculate PoD only for a particular failure mechanism, such as fiber breakage. Given the availability of signal classification methods (e.g. following the approach in $[9,18]$ ), the reference datasets may be reduced to finally hold only signals belonging to this mechanism. Then the resulting PoD calculation can be interpreted in terms of detectability of this failure mechanism only. Further, in many test cases, the test structure may not necessarily be loaded to final failure. With this in mind and the aspect that higher amplitude signals tend to appear in later stages towards failure, the reference datasets may also be limited until a certain load percentage to failure (e.g. up to $60 \%$ ultimate load). Consequently, the PoD calculation for the test structure are then more strictly representing the expected type of failure modes and amplitudes. This may 
result in stricter requirements for sensor placements in case the signal amplitudes are lower for these reference datasets.

\begin{tabular}{|c|c|}
\hline Strength & Weakness \\
\hline$\bullet$ & No model assumption of failure \\
probabilities required & Mix of different failure types and \\
- Mix of failure modes readily & datasets crucial for PoD values \\
available & Amplitude bias if no correction for \\
- If acquisition settings are identical in & confinement effect is made \\
reference case and test case, no & Need to generate $100 \%$ PoD \\
need to implement PoD changes & measurements at short distance and \\
due to equipment & keep acquisition settings constant \\
\end{tabular}

Table 2. Comparison of strengths and weaknesses of the approach using an experimentally obtained reference dataset.

\section{Acknowledgments}

We would like to thank Marvin A. Hamstad for the fruitful discussion of the presented approach and Andreea-Manuela Zelenyak for implementing the first steps of this PoD model as well as Philipp Potstada for the scientific discussion of the PoD measurements.

\section{Literature}

1. George A Georgiou: Probability of Detection (PoD) curves Derivation, applications and limitations., London (2006).

2. Mike Suits: NASA-STD-5009 Nondestructive Evaluation Requirements for Fracture Critical Metallic Components. (2008).

3. Sause, M., Hamstad, M.: Acoustic Emission Analysis. In: Beaumont, P.W.R. and Zweben, C.H. (eds.) Comprehensive Composite Materials II. pp. 291-326. Elsevier, Oxford (2018).

4. Sause, M.G.R., Haider, F., Horn, S.: Quantification of metallic coating failure on carbon fiber reinforced plastics using acoustic emission. Surf. Coatings Technol. 204, 300-308 (2009).

5. Hamstad, M.A., O'Gallagher, A., Gary, J.: A Wavelet Transform Applied To Acoustic Emission Signals: Part 1: Source Identification. J. Acoust. Emiss. 20, 39-61 (2002).

6. Hamstad, M.A., O'Gallagher, A., Gary, J.: A wavelet transform applied to acoustic emission signals: Part 2: Source location. J. Acoust. Emiss. 20, 62-82 (2002).

7. Pollock, A.: Probability of Detection for Acoustic Emission. 25, 167-172 (2007).

8. Pollock, A.A.: A PoD model for acoustic emission - discussion and status. In: Thompson, D.O. and Chimenti, D.E. (eds.) AIP Conference Proceedings 1211. pp. 1927-1933 (2010).

9. Sause, M.G.R.: In Situ Monitoring of Fiber-Reinforced Composites. Springer International Publishing, Cham (2016).

10. Grosse, C.U., Ohtsu, M.: Acoustic Emission Testing. Springer Berlin Heidelberg, Berlin, Heidelberg (2008).

11. Sause, M.G.R., Richler, S.: Finite Element Modelling of Cracks as Acoustic Emission Sources. J. Nondestruct. Eval. 34, 4 (2015). 
12. Cuadra, J., Vanniamparambil, P.A., Servansky, D., Bartoli, I., Kontsos, A.: Acoustic emission source modeling using a data-driven approach. J. Sound Vib. 341, 222-236 (2015).

13. Cuadra, J.A., Baxevanakis, K.P., Mazzotti, M., Bartoli, I., Kontsos, A.: Energy dissipation via acoustic emission in ductile crack initiation. Int. J. Fract. 199, 89-104 (2016).

14. Kharrat, M., Ramasso, E., Placet, V., Boubakar, L.: Acoustic Emission in Composite Materials under Fatigue Tests : Effect of Signal- Denoising Input Parameters on the Hits Detection and Data Clustering. In: 31st Conference of the European Working Group on Acoustic Emission. pp. 1-8. , Dresden, Germany (2014).

15. Hamstad, M.A., O'Gallagher, A., Gary, J.: Effects of Lateral Plate Dimensions on Acoustic Emission Signals from Dipole Sources. J. Acoust. Emiss. 19, 258-274 (2001).

16. Kalafat, S., Sause, M.G.R.: Acoustic emission source localization by artificial neural networks. Struct. Heal. Monit. 14, 633-647 (2015).

17. Sause, M.G.R., Müller, T., Horoschenkoff, A., Horn, S.: Quantification of failure mechanisms in mode-I loading of fiber reinforced plastics utilizing acoustic emission analysis. Compos. Sci. Technol. 72, 167-174 (2012).

18. Sause, M.G.R., Gribov, A., Unwin, A.R., Horn, S.: Pattern recognition approach to identify natural clusters of acoustic emission signals. Pattern Recognit. Lett. 33, 17-23 (2012).

19. Sause, M.G.R., Hamstad, M.A., Horn, S.: Finite element modeling of conical acoustic emission sensors and corresponding experiments. Sensors Actuators A Phys. 184, 6471 (2012). 